\title{
Positive contagion and the macrostructures of generalized balance
}

\author{
Noah E. Friedkin ${ }^{1 *}$ (D), Anton V. Proskurnikov ${ }^{2}$ and Francesco Bullo ${ }^{3}$ \\ ${ }^{1}$ Department of Sociology and the Center for Control, Dynamical Systems and Computation, University of California Santa \\ Barbara, Santa Barbara, CA, USA, ${ }^{2}$ Department of Electronics and Telecommunications, Politecnico di Torino, Turin, Italy \\ and the Institute for Problems of Mechanical Engineering, Russian Academy of Sciences, St. Petersburg, Russia (e-mail: \\ anton.p.1982@ieee.org) and ${ }^{3}$ Department of Mechanical Engineering and the Center for Control, Dynamical Systems and \\ Computation, University of California Santa Barbara, Santa Barbara, CA, USA (e-mail: bullo@engineering.ucsb.edu) \\ *Corresponding author. Email: friedkin@soc.ucsb.edu
}

Action Editor: Stanley Wasserman

\begin{abstract}
Balance theory has advanced with interdisciplinary contributions from social science, physical science, engineering, and mathematics. The common focus of attention is social networks in which every individual has either a positive or negative, cognitive or emotional, appraisal of every other individual. The current frontier of work on balance theory is a hunt for a dynamical model that predicts the temporal evolution of any such appraisal network to a particular structure in the complete set of balanced networks allowed by the theory. Finding such a model has proved to be a difficult problem. In this article, we contribute a parsimonious solution of the problem that explicates the conditions under which a network will evolve either to a set of mutually antagonistic cliques or to an asymmetric structure that allows agreement, cooperation, and compromise among cliques.
\end{abstract}

Keywords: balance theory, generalized balance, dynamical system

\section{Introduction}

The broad interest in balance theory is based on one of its special cases: a group in which every individual has a list of friends and enemies and belongs to one of two mutually antagonistic cliques, parties, or factions. If the group is a legislative body, then this special case corresponds to a two-party system in which the expectation of agreement, cooperation, and compromise is low, and legislative decisions depend more on the relative sizes of the two parties than on bipartisan deliberative processes. However, other special cases of the theory include networks with lower intensity positive and negative interpersonal appraisals in which multiple cliques, parties, or factions are connected in a structure of asymmetric positive appraisals that allow agreement, cooperation, and compromise. This article advances balance theory with a positive contagion model of the temporal evolution of any appraisal network to a particular structure in the complete set of balanced networks.

Balance theory considers a complete signed network with three or more individuals in which every individual $i$ has a positive or negative $\sigma_{i j}= \pm 1$ appraisal of every other individual $j$. In such networks every $(i, j)$ pair of individuals has two directed signed appraisals, $i \stackrel{ \pm}{\longrightarrow} j$ and $i \stackrel{ \pm}{\longleftarrow} j$. Thus, the theory assumes that perfect neutrality is impossible in any group of individuals whose behaviors are visible to every individual in the group. Since Cartwright and Harary's (1956) 


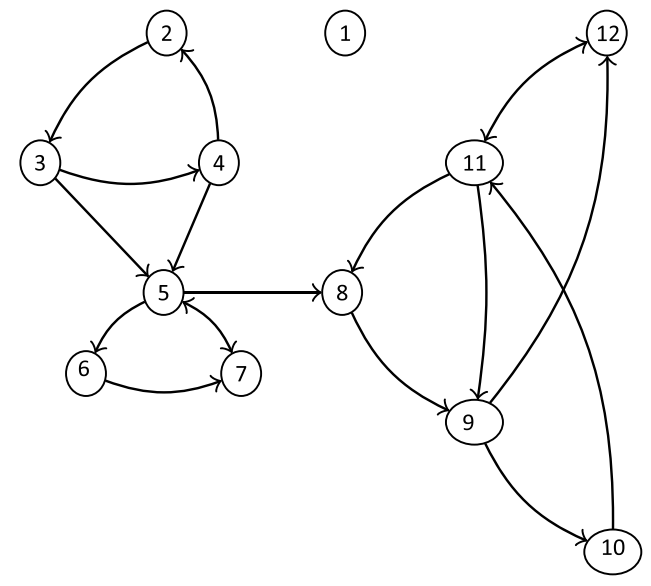

Figure 1. A directed graph of positive appraisals $\mathscr{G}$, corresponding to an appraisal network of $n=12$ individuals. Every $(i, j)$ pair of individuals has two directed signed appraisals, $i \stackrel{ \pm}{\longrightarrow} j$ and $i \stackrel{ \pm}{\longleftarrow} j$. The arcs of the graph are the individuals' set of positive appraisals (e.g., $2 \stackrel{+1}{\longrightarrow} 5$ ), and all other undisplayed arcs are negative appraisals (e.g., $2 \stackrel{-1}{\longrightarrow} 4,1 \stackrel{-1}{\longleftrightarrow} 2$ ).

formulation of the theory, there has been a sustained flow of interdisciplinary contributions to it. These contributions include empirical studies that have evaluated whether the theory's predictions are evident in observed appraisal networks (Davis, 1970, 1979; Davis \& Leinhardt, 1972; Facchetti et al., 2011; Hallinan, 1974; Holland \& Leinhardt, 1971; Ilany et al., 2013; Leskovec et al., 2010; Louch, 2000; Rawlings \& Friedkin, 2017), advancements of balance theory that have relaxed its assumptions (Davis, 1967, 1970; Easley \& Kleinberg, 2010; Harary et al., 1965; Johnsen, 1985; Montgomery, 2009), and studies of dynamical mechanisms that alter appraisal networks toward balance (Abell \& Ludwig, 2009; Antal et al., 2005, 2006; de Rijt, 2011; Hummon \& Doreian, 2003; Jia et al., 2016; Kułakowski et al., 2005; Marvel et al., 2011; Sørensen \& Hallinan, 1976; Srinivasan, 2011; Traag et al., 2013).

The paper is organized as follows. First, we briefly review Cartwright and Harary's seminal formalization of balance (Section 2), the current state of work on its generalization (Section 3), and the current state of work on related dynamical mechanisms (Section 4). After introducing some graph-theoretic preliminaries in Section 5, we introduce our positive contagion mechanism (Section 6) and analyze its implications for the suite of macrostructures allowed by it (Section 7). We conclude with a discussion of the open theoretical problems that our model has addressed, the distinctive properties of the model, and their implications (Section 8).

\section{Classic balance}

The focal special case of a balanced network of positive and negative interpersonal appraisals is a network of extremal appraisals governed by four rules. (1) An enemy of an enemy is a friend, that is, if $i \stackrel{-1}{\longrightarrow} k$ and $k \stackrel{-1}{\longrightarrow} j$, then $i \stackrel{+1}{\longrightarrow} j$. (2) A friend of an enemy is an enemy, that is, if $i \stackrel{-1}{\longrightarrow} k$ and $k \stackrel{+1}{\longrightarrow} j$, then $i \stackrel{-1}{\longrightarrow} j$. (3) An enemy of a friend is an enemy, that is, if $i \stackrel{+1}{\longrightarrow} k$ and

$k \stackrel{-1}{\longrightarrow} j$, then $i \stackrel{-1}{\longrightarrow} j$. (4) A friend of a friend is a friend, that is, if $i \stackrel{+1}{\longrightarrow} k$ and $k \stackrel{+1}{\longrightarrow} j$, then $i \stackrel{+1}{\longrightarrow} j$. Rule 4, henceforth referred to as transitivity, has a special status. It says that $i$ will agree with the positive appraisals of $k$ if $i$ positively appraises $k$. Hence, any subgraph of the network in which every $(i, j)$ pair of individuals in the subgraph has a path of positive appraisals from $i$ to $j$ and a path of positive appraisals from $j$ to $i$ must be a clique with all positive within-clique appraisals, whose members agree in their positive or negative appraisals of all other members of network. In general, a complete signed network is uniquely determined by its directed graph $\mathscr{G}$ of positive appraisals as in Figure 1 in which the absence of an $i$ to $j$ arc implies the existence of a negative $i$ to $j$ arc. The Figure 1 network includes instances of violations of all four rules. It can be shown that in a complete signed network, any triple $i \neq j \neq k$ must be one of the 16 types of configurations of positive appraisals as in Figure 2, and that 14 of these possible types violate one or 


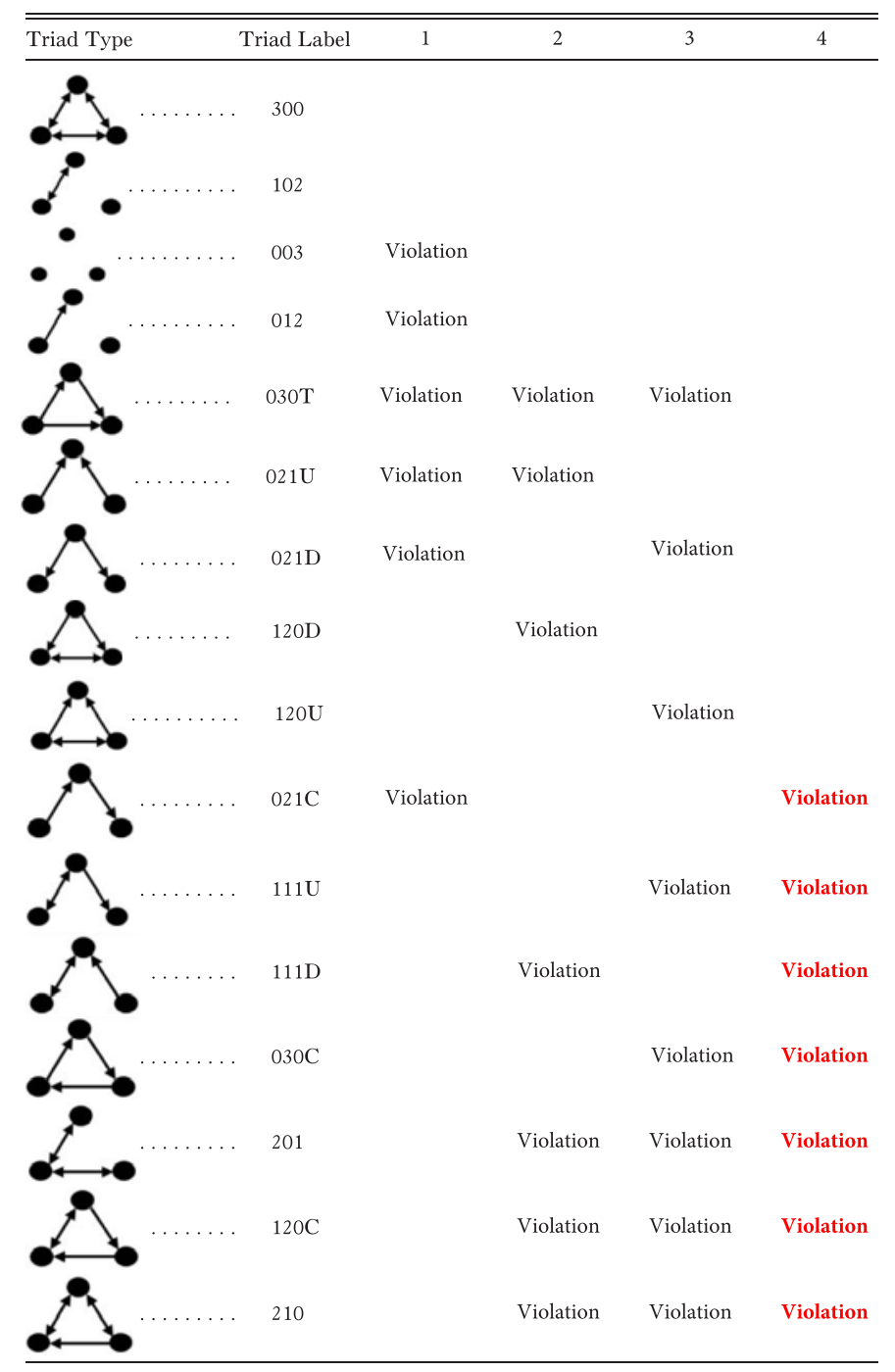

Figure 2. Four rules of classic balance and the triads violating them (the arcs stand for positive appraisals). Rule 1: An enemy of an enemy is a friend. Rule 2: A friend of an enemy is an enemy. Rule 3: An enemy of a friend is an enemy. Rule 4: A friend of a friend is a friend. Classic balance allows no violations of rules 1-4. Generalized balance allows no violations of rule 4 and includes classic balance as special case. The seven triads with violations of rule 4 (if $i \stackrel{+1}{\longrightarrow} k$ and $k \stackrel{+1}{\longrightarrow} j$, then $i \stackrel{+1}{\longrightarrow} j$ ) cannot appear in any balanced appraisal network.

more of rules $1-4$. The two triad types permitted by these four rules are composed of symmetric appraisals, and any network strictly composed of these two triad types satisfies $\sigma_{i k} \sigma_{k j} \sigma_{i j}=+1$ for any triple $i \neq j \neq k$. It can be shown that this special case implies a network in which any cyclic sequence $i_{1} \rightarrow i_{2} \rightarrow \ldots \rightarrow i_{m} \rightarrow i_{1}$ contains an even number of negative appraisals, that is, $\sigma_{i_{1} i_{2}} \sigma_{i_{2} i_{3}} \ldots \sigma_{i_{m} i_{1}}=+1$. Such an appraisal network must be either (i) a network of all positive appraisals or (ii) a network that is partitioned into two cliques of individuals with all positive within-clique appraisals and all negative between-clique appraisals. Classic balance theory predicts that any appraisal network, such as Figure 1, will evolve either to one clique or two cliques with all positive within-clique appraisals and all negative between-clique appraisals. This fundamental result was discovered by Cartwright and Harary (1956), whose formalization of Heider's works (Heider, 1944, 1946) opened the field of balance theory. 

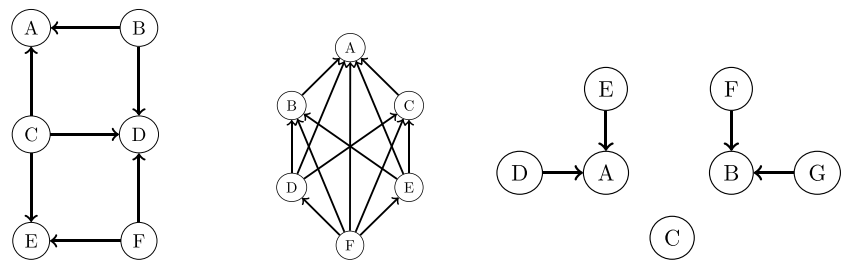

(C)

Figure 3. The displayed graphs are special cases of generalized balance theory. The nodes A, B, . are cliques of individuals. Each clique is either a nontrivial clique of multiple individuals with all positive interpersonal appraisals, or a trivial clique with one individual. The $\rightarrow$ arcs between two cliques $(U, V)$ is an asymmetric relation in which all U's appraisals of $\mathrm{V}$ are positive and all V's appraisals of $U$ are negative. The absence of an arc between two cliques stands for all negative between-clique appraisals.

\section{Generalized balance}

Generalized balance theory is a response to empirical evidence on classic balance predictions (Davis, 1970, 1979; Davis \& Leinhardt, 1972; Hallinan, 1974; Holland \& Leinhardt, 1971; Rawlings \& Friedkin, 2017). Classic balance theory predicts one or two mutually antagonistic cliques. The evidence instead documents the prevalence of three or more cliques and positive asymmetric connectivity among cliques. The empirical evidence also shows that appraisal network evolution is not reliably governed by rules 1-3 and instead is mainly based on the elimination of violations of transitivity. These findings triggered the development of generalized balance theory, which allows all nine types of triads that do not violate transitivity in contrast to classic balance, which is constrained to two types of triads. In generalized balance theory, a network $\mathscr{G}$ is structurally balanced if it does not violate transitivity. It predicts that a network with violations of transitivity will evolve to a network in which all violations of transitivity are eliminated. The extremal terms "friend" and "enemy" are also relaxed to allow signed relations with heterogeneous levels of positive and negative appraisals; that is, an $i \stackrel{ \pm}{\longrightarrow} j$ may correspond to lower intensity positive or negative appraisals. Generalized balance theory's domain of special cases includes any $\mathscr{G}$ composed of a subset of the nine triad types that do not violate transitivity. Figure 3 illustrates several of such special cases. In generalized balance theory, the topology of balanced (transitive) appraisal networks may or may not be constrained by any shared rules on negative appraisals. Special cases of networks in which one, two, or three of the rules 1-3 are satisfied might either be based on the postulate of a shared group interpersonal appraisal culture, or on the postulate that such special cases are epiphenomena of the mechanism that eliminates violations of transitivity. In this article we develop the latter postulate.

\section{Dynamical mechanisms}

Modeling of the dynamical mechanisms, which evolve networks to a state of structural balance, has mainly focused on mechanisms that generate the classic Cartwright and Harary (1956) special case. As Marvel et al. (2011) remark, finding a mechanism that generates this special case has proved to be a challenge. Following Kułakowski et al. (2005), a line of work has been advanced on this special case under the simplifying assumption of symmetric networks (Antal et al., 2006; Marvel et al., 2011; Srinivasan, 2011; Zheng et al., 2014). With this assumption, the number of possible triad types is reduced from 16 to 4 types $(300,102,003,201)$, and the problem simplifies to a hunt for a mechanism that eliminates 003 and 201 types of triads. In classic balance, symmetric positive appraisal is an emergent condition of the resolution of violations of transitivity under the constraints of rules 1-3. In generalized balance, where the constraints of rules 1-3 are relaxed, symmetric positive appraisals are restricted to cliques that may be connected by asymmetric appraisals. Thus, we discard the symmetry assumption. The dynamical problem posed by generalized balance is to find a natural mechanism of individuals' temporal alterations of their own appraisals leading to a stable network of appraisals. This mechanism should (i) satisfy the 
condition of transitivity and (ii) predict what class of balanced condensed macrostructure an appraisal network will evolve to (e.g., the classic Cartwright-Harary macrostructure, or one of the Figure 3 macrostructures, or some other form). The solution of the problem is complex when the postulated mechanism allows conversions of positive appraisals to negative appraisals.

We replace the simplifying structural assumption of a symmetric unbalanced $\mathscr{G}$ with the simplifying assumption of a positive contagion (transitive closure) mechanism that eliminates violations of transitivity. The postulate of this mechanism is motivated by empirical evidence that conversions of appraisals mainly propagate positive appraisals that eliminate violations of transitivity (Davis, 1970; Davis \& Leinhardt, 1972; Hallinan, 1974; Holland \& Leinhardt, 1971; Louch, 2000; Rawlings \& Friedkin, 2017). The mechanism transforms any unbalanced appraisal network of $n \geq 3$ individuals to a stable balanced network in $n-2$ steps. It is an influence system mechanism that maintains positive appraisals and resolves appraisal disagreements, thus forming cliques of individuals who agree in their appraisals of all members of the network. As we will show, an attractive implication of this mechanism is that graph theory's four topological categories of networks (strong, unilateral, weak, and disconnected) are categorical distinctions that transfer to the class of balanced condensed macrostructures generated by it, and that each such class is associated with a distinctive set of allowed triads. In this framework, the focal Harary and Cartwright (1956) case of classic balance evolves from a disconnected $\mathscr{G}$ with two strong components. In the next section, we introduce the relevant concepts.

\section{Graph-theoretic preliminaries}

Consider a directed graph (network) $\mathscr{G}$ of $n$ nodes where some ordered pairs $(i, j)$ of nodes are connected by arcs $i \rightarrow j$. The structure of arcs is encoded by the graph's binary adjacency matrix $\mathbf{X}=\left(x_{i j}\right)$, where $x_{i j}=1$ if the $i \rightarrow j$ arc exists, and otherwise $x_{i j}=0$. A subgroup of nodes and all arcs between them constitutes a (induced) subgraph $\mathscr{G}^{\prime}$ of $\mathscr{G}$. Subgraphs correspond to submatrices $\mathbf{X}^{\prime}=\left(x_{i j}\right)_{i, j \in V^{\prime}}$ of $\mathbf{X}$, where $V^{\prime} \subseteq\{1, \ldots, n\}$ is a subset of nodes. Henceforth, unless otherwise stated, we assume reflexive $\mathscr{G}$ with $i \rightarrow i$ arcs (loops), that is, $x_{i i}=1$ for all $i$. In our figures, these loops will be omitted. Nodes $i$ and $j$ are structurally equivalent if $x_{i j}=x_{j i}, x_{i k}=x_{j k} \forall k \neq i, j$. If $\mathscr{G}$ is the graph of positive appraisals of a complete signed network, then a set of two or more structurally equivalent nodes is a set of individuals with positive self-appraisals, who agree in the signs of each other's appraisals and their appraisals of all other individuals.

A sequence of arcs $i \rightarrow k_{1} \rightarrow \ldots k_{s-1} \rightarrow j$ connecting $i$ to $j$ is said to be a walk of length $s \geq 1$, connecting $i$ to $j$. A walk is a path if no node in it appears more than once $\left(k_{1}, \ldots, k_{s}, j\right.$ are all different), and it is a cycle if $j=i$. An $\operatorname{arc} i \rightarrow j$ is a path of length 1 from $i$ to $j$, and a loop $i \rightarrow i$ is a trivial cycle. A graph is acyclic if it contains no cycles but for the self-loops.

A node $j$ is reachable from $i$ if at least one path from $i$ to $j$ exists; if $i$ is also reachable from $j$, then $i$ and $j$ are mutually reachable. A graph is strong (strongly connected) if any two of its nodes are mutually reachable. A strong component of a graph is a maximal strong subgraph, that is, no node can be added to it without destroying its strong connectivity. A strong graph contains only one strong component (the whole graph). A clique in a graph is a subgraph, which is complete (i.e., any two nodes $i, j$ in it are mutually connected $i \leftrightarrow j$ ). Note that a clique is a strong component if the clique is maximal. Cliques and components can be trivial, containing only a single node; otherwise they are said to be nontrivial. In an acyclic graph, every node is both a trivial clique and trivial strong component. Figure 4 illustrates the strong components of the graph shown in Figure 1.

Given an adjacency matrix $\mathbf{X}$, its symmetrization $\mathbf{X}^{\dagger}=\left(x_{i j}^{\dagger}\right)$ consists of the entries $x_{i j}^{\dagger}=x_{j i}^{\dagger}=$ $\max \left(x_{i j}, x_{j i}\right)$. The corresponding graph $\mathscr{G}^{\dagger}$ is referred to as the symmetrization of $\mathscr{G}$; walks in $\mathscr{G}^{\dagger}$ are called semi-walks in $\mathscr{G}$. Graph $\mathscr{G}$ is connected if $\mathscr{G}^{\dagger}$ is strong, that is, each pair of nodes $(i, j)$ is connected by a semi-walk. Otherwise, $\mathscr{G}$ is said to be disconnected.

Any $\mathscr{G}$ belongs to one of four connectivity categories-strong, unilateral, weak, or disconnected. Strong connectivity has been already defined: it requires mutual reachability for any pair 


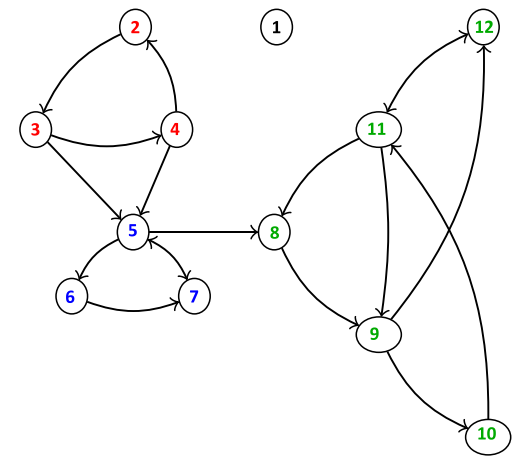

Strong Components

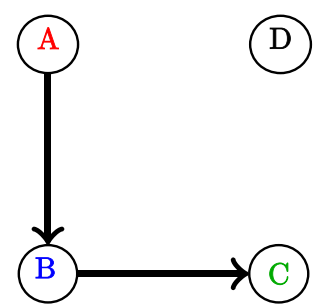

Condensed Macrostructure

Figure 4. The four strong components of the Figure 1 network $\mathscr{G}$. Its nontrivial components are $A=\{2,3,4\}, B=\{5,6,7\}$, and $C=\{8,9,10,11,12\}$, and its trivial component is $D=\{1\} . A-C$ are not cliques, and $D$ is a trivial clique. The condensed macrostructure of the network is an acyclic graph with four nodes $(A, B, C, D)$ corresponding to the four strong components, and two $\operatorname{arcs} A \rightarrow B$ and $B \rightarrow C$ indicating the existence of least arc from $A$ to $B$ (there are two such arcs), and at least one arc from $B$ to $C$ (there is one such $\operatorname{arc}$ ).

of nodes $i$ and $j$. If $\mathscr{G}$ is not strong, then it is unilateral (unilaterally connected) if either $i$ can reach $j$ or $j$ can reach $i$ in all $(i, j)$ pairs of nodes. A $\mathscr{G}$ is weak (weakly connected) if it is connected, but not unilateral. If $\mathscr{G}$ is not connected, then it is said to be disconnected.

Given a directed graph $\mathscr{G}$, its condensed macrostructure (or condensation) $\mathscr{G}^{*}$ is a directed graph whose nodes are strong components of $\mathscr{G}$. An arc in $\mathscr{G}^{*}$ exists between strong components $U$ and $V$ if some members $i \in U$ and $j \in V$ are connected by arc $i \rightarrow j$ in $\mathscr{G}$. In $\mathscr{G}^{*}$, there may be one or multiple nodes, and no or some asymmetric arcs; the graph $\mathscr{G}^{*}$ is always acyclic, in particular, mutual connections $U \leftrightarrow V$ are impossible. The nodes of any acyclic directed graph (in particular, the condensation $\mathscr{G}^{*}$ ) can always be reordered in such a way that from node $i$ only itself and nodes with greater indices $j>i$ can be reached. Such a reordering renders the adjacency matrix $\mathbf{X}^{*}$ upper-diagonal. The condensed macrostructure of a graph inherits its connectedness category.

Lemma 1. (Harary et al., 1965, Theorem 3.4) The connectivity types of $\mathscr{G}$ and $\mathscr{G}^{*}$ (strong, unilateral, weak, or disconnected) coincide.

Using induction on $s$, it can be shown that the matrices $\mathbf{X}^{s}$, where $s=1,2, \ldots$, encode $\mathscr{G}$ 's walks of length $s$, that is, $\left(\mathbf{X}^{s}\right)_{i j}>0$ if and only if a walk of length $s$ from $i$ to $j$ exists in $\mathscr{G}$. Obviously, if $j$ is reachable from $i$, the shortest path from $i$ to $j$ has length no more than $n-1$. Since each node has a self-loop, each walk of length $s<n-1$ can be augmented to a walk of length $n-1$. For this reason, $\mathbf{X}^{n-1}$ may be considered as the reachability matrix of the graph: $\left(\mathbf{X}^{n-1}\right)_{i j}>0$ if and only if $j$ is reachable from $i$.

Definition 1. Let $\mathbf{X}$ be $n \times n$ adjacency matrix of some graph $\mathscr{G}$ and $x_{i i}=1$ for all $i$. The graph corresponding to the reachability matrix $\mathbf{X}^{n-1}$ is the transitive closure of $\mathscr{G}$ and is denoted by $\overline{\mathscr{G}}$. In other words, $i \rightarrow j$ in $\overline{\mathscr{G}}$ if and only if $j$ is reachable from in $\mathcal{G}$.

Definition 2. Following Harary et al. (1965), the graph $\mathscr{G}$ is transitive if it contains arc $i \rightarrow j$ whenever two arcs $i \rightarrow k$ and $k \rightarrow j$ exist for some $k \neq i, j$.

Using induction on $s=1,2, \ldots$, it can be proved that if $i$ and $j$ are connected by a walk of length $s \geq 1$, then transitivity automatically implies the existence of arc $i \rightarrow j$. Thus, any strong component of a transitive graph is a clique. Furthermore, if $i$ and $j$ belong to the same clique (and thus mutually connected $i \leftrightarrow j$ ), then they are structurally equivalent: the existence of arc 
$i \rightarrow k$ implies the existence of $j \rightarrow k$ and vice versa. Finally, it can be shown that if $\mathscr{G}$ is transitive, the same holds for its condensed macrostructure $\mathscr{G}^{*}$. It appears that the latter properties (transitive condensed macrostructures composed of strong components that are cliques of structurally equivalent nodes) in turn imply transitivity of $\mathscr{G}$. We summarize these facts in a key lemma that follows from standard results of graph theory (Harary et al., 1965, Chapter 5).

Lemma 2. The following statements are equivalent: (i) graph $\mathscr{G}$ is transitive; (ii) $\overline{\mathscr{G}}=\mathscr{G}$, that is, $j$ is reachable from in $\mathscr{G}$ if and only if there is an arc $i \rightarrow j$; (iii) all strong components of $\mathscr{G}$ are cliques, the nodes of each nontrivial clique are structurally equivalent, and the condensed macrostructure $\mathscr{G}^{*}$ is transitive. Among all graphs containing $\mathscr{G}$ as a subgraph, $\overline{\mathscr{G}}$ is the minimal transitive graph, that is, no arc can be removed from $\overline{\mathscr{G}}$ without destroying transitivity. The graphs $\mathscr{G}$ and $\mathscr{G}$ have same strong components, and $(\overline{\mathscr{G}})^{*}=\overline{\mathscr{G}^{*}}$.

Lemmas 1 and 2 imply the following inheritance corollaries: The connectivity category of the graph $\mathscr{G}$ (strong, unilateral, weak, or disconnected), its transitive closure $\overline{\mathscr{G}}$, and its condensation $\mathscr{G}^{*}$ are identical. If the graph of positive appraisals $\mathscr{G}$ is strong (a single strong component), then transitive closure generates a network that is one clique of structurally equivalent individuals who positively appraise each other. In the more interesting case of $G$ with two or more strong components, transitive closure transforms the strong components into cliques and generates a network in which every $(U, V)$ pair of cliques has either a symmetric $N^{*}$ negative or asymmetric $A^{*}$ relation

$$
(U, V)= \begin{cases}\mathrm{N}^{*}, & \text { if } \forall i \in U, i \stackrel{-1}{\longrightarrow} j, \forall j \in V, \text { and } \forall j \in V, j \stackrel{-1}{\longrightarrow} i, \forall i \in U, \\ \mathrm{~A}^{*}, & \text { if } \forall i \in U, i \stackrel{+1}{\longrightarrow} j, \forall j \in V \text { and } \forall j \in V, j \stackrel{-1}{\longrightarrow} i, \forall i \in U .\end{cases}
$$

For the Figure 1 non-transitive graph of positive appraisals $\mathscr{G}$, the following tabulation illustrates the adjacency matrices related to its transitive closure (Definition 1) $\overline{\mathscr{G}}$ :

$$
\mathbf{X}=\left[\begin{array}{l|lll|lll|lllll}
1 & 0 & 0 & 0 & 0 & 0 & 0 & 0 & 0 & 0 & 0 & 0 \\
\hline 0 & 1 & 1 & 0 & 0 & 0 & 0 & 0 & 0 & 0 & 0 & 0 \\
0 & 0 & 1 & 1 & 1 & 0 & 0 & 0 & 0 & 0 & 0 & 0 \\
0 & 1 & 0 & 1 & 1 & 0 & 0 & 0 & 0 & 0 & 0 & 0 \\
\hline 0 & 0 & 0 & 0 & 1 & 1 & 1 & 1 & 0 & 0 & 0 & 0 \\
0 & 0 & 0 & 0 & 0 & 1 & 1 & 0 & 0 & 0 & 0 & 0 \\
0 & 0 & 0 & 0 & 1 & 0 & 1 & 0 & 0 & 0 & 0 & 0 \\
\hline 0 & 0 & 0 & 0 & 0 & 0 & 0 & 1 & 1 & 0 & 0 & 0 \\
0 & 0 & 0 & 0 & 0 & 0 & 0 & 0 & 1 & 1 & 0 & 1 \\
0 & 0 & 0 & 0 & 0 & 0 & 0 & 0 & 0 & 1 & 1 & 0 \\
0 & 0 & 0 & 0 & 0 & 0 & 0 & 0 & 1 & 0 & 1 & 1 \\
0 & 0 & 0 & 0 & 0 & 0 & 0 & 0 & 0 & 0 & 1 & 1
\end{array}\right], \quad\left[\begin{array}{lllll|lll|lllll}
1 & 0 & 0 & 0 & 0 & 0 & 0 & 0 & 0 & 0 & 0 & 0 \\
\hline 0 & 1 & 1 & 1 & 1 & 1 & 1 & 1 & 1 & 1 & 1 & 1 \\
0 & 1 & 1 & 1 & 1 & 1 & 1 & 1 & 1 & 1 & 1 & 1 \\
0 & 1 & 1 & 1 & 1 & 1 & 1 & 1 & 1 & 1 & 1 & 1 \\
\hline 0 & 0 & 0 & 0 & 1 & 1 & 1 & 1 & 1 & 1 & 1 & 1 \\
0 & 0 & 0 & 0 & 1 & 1 & 1 & 1 & 1 & 1 & 1 & 1 \\
0 & 0 & 0 & 0 & 1 & 1 & 1 & 1 & 1 & 1 & 1 & 1 \\
\hline 0 & 0 & 0 & 0 & 0 & 0 & 0 & 1 & 1 & 1 & 1 & 1 \\
0 & 0 & 0 & 0 & 0 & 0 & 0 & 1 & 1 & 1 & 1 & 1 \\
0 & 0 & 0 & 0 & 0 & 0 & 0 & 1 & 1 & 1 & 1 & 1 \\
0 & 0 & 0 & 0 & 0 & 0 & 0 & 1 & 1 & 1 & 1 & 1 \\
0 & 0 & 0 & 0 & 0 & 0 & 0 & 0 & 1 & 1 & 1 & 1 & 1
\end{array}\right],
$$


The adjacency matrix $\mathbf{X}$ associated with the Figure 1 graph $\mathscr{G}$ becomes the adjacency matrix $\overline{\mathbf{X}}$ associated with its transitive closure $\overline{\mathscr{G}}$ : (i) each strong component of $\mathscr{G}$ corresponds to a complete strong component (clique) in $\overline{\mathscr{G}}$, (ii) any incomplete set of positive appraisals from a strong component $\mathrm{U}$ to strong component $\mathrm{V}$ corresponds to a complete $\mathrm{A}^{*}$ relation if and only if there exists at least one arc $i \in U \rightarrow j \in V$, and (iii) all violations of transitivity are eliminated. The weak connectivity of $\overline{\mathscr{G}}$ is an inherited trait of $\mathscr{G}$. The adjacency matrices associated with the macrostructures of $\mathscr{G}$ and $\overline{\mathscr{G}}$ are identical $\mathbf{X}^{*}=\overline{\mathbf{X}}^{*}$. Note that the transitive closure of Figure 1 results in a network with a near complete agreement of positive appraisals of one clique.

\section{Positive contagion}

In generalized balance theory, a complete signed network is structurally balanced if its graph of positive appraisals $\mathscr{G}$ is transitive. The nine triad types that do not violate transitivity include triads that do not contain any path $i \rightarrow k \rightarrow j$. Such triads are said to be vacuously transitive. Ongoing chaotic changes of signs, in general, do not result in a stable balanced network without violations of transitivity. In contrast, all such violations will be rapidly resolved if $\stackrel{+1}{\longrightarrow}$ arcs are stable and a contagion mechanism converts $i \stackrel{-1}{\longrightarrow} j$ arcs to $i \stackrel{+1}{\longrightarrow} j$ arcs if at least one $k \neq i, j$ exists such that $i \stackrel{+1}{\longrightarrow} k \stackrel{+1}{\longrightarrow} j$. With each such conversion, $i$ resolves an appraisal disagreement with the corresponding $k$. It also may introduce new violations of transitivity, but since the total number of arcs in the graph of positive appraisal is limited, the process of conversion terminates in finite time (no more than $\leq n-2$ steps) and, as will be shown, the terminal graph coincides with the transitive closure of the initial positive appraisal network. We refer this mechanism as positive contagion. It is a mechanism that rapidly builds (i) maximal cliques of individuals with enduring positive relationships that, in sociology and anthropology, have long been emphasized as the "primary" social units of social cohesion, social control, and social support (Friedkin, 2004; Shils, 1951), and (ii) the asymmetric hierarchical networks noted by Davis and Leinhardt (1972) that triggered the development of generalized balance theory. Lemma 2 implies that such a mechanism preserves the connectivity category of the network's condensed macrostructure during the evolution of the network toward generalized structural balance. All special cases of generalized balance are covered by this mechanism.

We now develop a mechanism that attends to the individual decision-making involved in an $i$ 's conversion of an appraisal $i \stackrel{-1}{\longrightarrow} j$ into $i \stackrel{+1}{\longrightarrow} j$, adding thus a new arc into the positive appraisal graph $\mathscr{G}$. The assumption of positive appraisal stability implies that the decision to convert a negative appraisal to positive appraisal is irreversible, that is, $\mathscr{G}$ can only acquire new arcs, but cannot lose them. This assumption comports with empirical evidence that conversions of negative arcs to positive arcs are more typical than "reversed" conversions from positive arcs into negative ones, and that the temporal reduction of violations of transitivity appears to be the main moving part of appraisal network evolution (Rawlings \& Friedkin, 2017).

Along with the standard binary adjacency matrix of a graph, one may consider a more general weighted adjacency matrix $\mathbf{X}=\left(x_{i j}\right)$, where $x_{i j} \in(0,1]$ if arc $i \rightarrow j$ exists and $x_{i j}=0$ otherwise. Dealing with a positive appraisal graph, the weight $x_{i j}$ can be interpreted as the appraisal's strength. A pair $(\mathscr{G}, \mathbf{X})$ is said to be a weighted (or valued) graph. We call two weighted adjacency matrices $\mathbf{X}, \tilde{\mathbf{X}}$ equivalent (denoted $\mathbf{X} \sim \tilde{\mathbf{X}}$ ) if they correspond to the same graph $\mathscr{G}$, that is, $x_{i j}>0$ if and only if $\tilde{x}_{i j}>0$. Similarly, one can consider a weighted signed complete appraisal network $\mathscr{S}$, whose signed adjacency matrix $\mathbf{S}=\left(s_{i j}\right)_{i, j}, s_{i j} \in[-1,1] \backslash\{0\}$, encodes positive $\left(s_{i j}>0\right)$ and negative $\left(s_{i j}<\right.$ 0 ) appraisals of heterogeneous strengths. We say that the weighted graph $(\mathscr{G}, \mathbf{X})$ is the graph of positive appraisals of the weighted signed network $(\mathscr{S}, \mathbf{S})$ if $x_{i j}=\max \left(0, s_{i j}\right)$. It is always assumed that $s_{i i}=x_{i i}>0 \forall i$, that is, individuals appraise themselves positively. In this situation, $\mathbf{X}^{n-1}$ is a (weighted) adjacency matrix of the transitive closure $\overline{\mathscr{G}}$. 
The process of positive contagion evolves the weighted graph of positive appraisals $(\mathscr{G}(t), \mathbf{X}(t))$, where $t=1,2, \ldots$. During this process, $\mathscr{G}(t)$ can acquire (but not lose) arcs, and the weights on arcs can be altered. In terms of the signed network $\mathbf{S}(t)$, the evolution switches negative appraisals $i \stackrel{s_{i j}(t)<0}{\longrightarrow} j$ to positive ones $i \stackrel{s_{i j}(t+1)>0}{\longrightarrow} j$ and alters the strengths of the positive appraisals. An evolution of negative appraisals, except for the aforementioned switchings, does not play any role.

Let $\mathbf{X}(1)$ be the weighted adjacency matrix of a positive appraisal graph $\mathscr{G}$ and $\mathbf{W} \sim \mathbf{X}(1)$ be row-stochastic, that is, $0 \leq w_{i j} \leq 1, \sum_{j=1}^{n} w_{i j}=1 \forall i$. Then the DeGroot (1974) influence system dynamics

$$
\mathbf{X}(t+1)=\mathbf{W} \mathbf{X}(t)=\ldots=\mathbf{W}^{t} \mathbf{X}(1), \quad t=1,2, \ldots
$$

is a positive contagion mechanism that generates an evolving adjacency matrix based on the following simple rule. If individual $i$ s initial appraisal of $j$ is positive $\left(x_{i j}(1)>0 \Leftrightarrow w_{i j}>0\right)$ and $i$ disagrees with $j$ 's positive appraisal of some other individual $k \neq i, j, x_{i k}(t)=0<x_{j k}(t)$, then $i$ resolves this disagreement by converting the negative appraisal of $k$ to a positive appraisal at the next period $x_{i k}(t+1) \geq w_{i j} x_{j k}(t)>0$. These conversions of appraisals from negative to positive are irreversible: if $x_{i j}(t)>0$, then $x_{i j}(t+1) \geq w_{i i} x_{i j}(t)>0$. Using induction on $s$, it can be shown that $\mathbf{X}(s) \sim \mathbf{X}(1)^{s}$ and hence $\mathbf{X}(n-1)$ stands for the weighted adjacency matrix of the transitive closure $\overline{\mathscr{G}}$. In other words, the mechanism eliminates any violation of transitivity and achieves the transitive closure of $\mathscr{G}$ in at most $n-2$ iterations. After this, the topology encoded by the adjacency matrix $\mathbf{X}(t)$ remains unchanged (while the strengths of appraisals continue evolving).

The assumption of a time-invariant $\mathbf{W}$ in (2) may be relaxed in two ways. (i) While maintaining the assumption $w_{i j}>0$ if $x_{i j}(1)=1$ and $w_{i j}=0$ if $x_{i j}(1)=0$, one can relax the assumption of timeinvariance by allowing changes in the values $w_{i j}$ along the sequence $t=2,3, \ldots$ (ii) The more interesting relaxation assumes that $\mathbf{W}(t) \sim \mathbf{X}(t)$, that is, $\mathbf{W}$ is altered with temporal propagation of new positive appraisals:

$$
\mathbf{X}(t+1)=\mathbf{W}(t) \mathbf{X}(t)
$$

Updating the set of positive $w_{i j}(t)$ accelerates the process of transitive closure computation. The topology of the graph $\mathscr{G}(t)$ coincides with the closure $\overline{\mathscr{G}}(1)$ after at most $1+\left\lceil\log _{2}(n-1)\right\rceil$ steps since, as can be easily shown, $\mathbf{X}(s) \sim \mathbf{X}(1)^{2^{s-1}}$. In algorithmic graph theory, an equivalent procedure to compute the transitive closure $\mathbf{X}(t+1)=\mathbf{X}(t)^{2}$ is referred to as the Warshall algorithm (Warshall, 1962).

\section{Classes of balanced condensed macrostructures}

With positive contagion particular structural conditions of the initial appraisal network $\mathscr{G}$ predict the structure of the transitive closure and, in turn, the structure of the transitive closure is uniquely determined by $\mathscr{G}^{\prime}$ s condensed macrostructure $\mathscr{G}^{*}=\overline{\mathscr{G}}^{*}$. If the graph is strongly connected, then it has one strong component, and its condensed macrostructure is single node. If the graph is connected but not strongly connected, then it contains multiple strong components and its condensed macrostructure must be either a unilateral or a weak acyclic graph, and thus must include at least one sink and least one source nodes. Sinks of $\mathscr{G}^{*}$ stand for strong components in $\mathscr{G}$ with one or more incoming arcs and no outgoing arcs; sources stand for strong components with one or more outgoing arcs and no incoming arcs. Each category of a connected $\mathscr{G}$ (strong, unilateral, and weak) permits a distinctive subset of appraisal configurations among any three individuals.

Strong Condensed Macrostructures. The condensed macrostructure $\mathscr{G}^{*}$ of a strong graph $\mathscr{G}$ is a singleton, and its transitive closure $\overline{\mathscr{G}}$ is a single clique. The set of its permitted triads is $\mathfrak{S}=\{300\}$.

Unilateral Condensed Macrostructures. If the graph $\mathscr{G}$ is unilateral, then it contains two or more strong components that include (i) a unique source component, (ii) a unique sink component, and (iii) a unique complete (Hamiltonian) path that starts from the source component and includes 
(a)

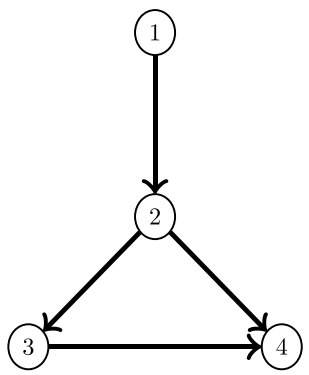

Condensed Macrostructure $\mathscr{G}^{*}$ of $\mathscr{G}$ (b)

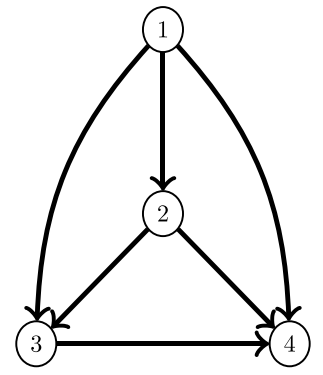

Condensed Macrostructure $\overline{\mathscr{G}} *$ of $\overline{\mathscr{G}}$

Figure 5. (a) The condensed macrostructure $\mathscr{G}^{*}$ of a unilateral $\mathscr{G}$ with four strong components. (b) Transitive closure transforms any incomplete nontrivial strong component of $\mathscr{G}$ into a clique, transforms any positive relation between two strong components into a complete asymmetric $A^{*}$ relation, and eliminates any violation of transitivity.

all other components (Harary et al., 1965, Theorem 3.10). Note that the Figure 3 network that has a unique source and a unique sink is not a unilateral network. Figure 5 is an example of the condensed macrostructure $\mathscr{G}^{*}$ of a unilateral $\mathscr{G}$, and its transitive closure $\overline{\mathscr{G}}^{*}$. Without loss of generality, we may reorder the components in a way that this complete path is $1 \rightarrow 2 \rightarrow \ldots \rightarrow m$, where component 1 is the source and component $m$ is the sink. Then component $s$ is connected to all components $r>s$. Compared to the case of strong network, three more types of triads are possible in a transitive unilateral graph. Any three nodes $i \neq j \neq k$ can belong to a single cliquecomponent (triad 300), three different cliques (triad 030T), or two cliques, for example, $i, j$ belong to one clique and $k$ to the other clique (constituting triad $120 \mathrm{D}$ or $120 \mathrm{U}$ ). Therefore, the set of permitted triads in transitive unilateral networks is $\mathfrak{U}=\mathfrak{S} \cup\{120 D, 120 U, 030 T\}$.

Weak Condensed Macrostructures. If the graph $\mathscr{G}$ is weak, then its transitive condensed macrostructure $\mathscr{G}^{*}$ may include multiple sink and source cliques. If at least one of these cliques is nontrivial, say $U$, then the graph contains a triad 102 (constituted by two nodes from $U$ and a node from some other component $V$ ). Besides this, a weak graph can contain all the remaining triads that do not violate transitivity $\mathfrak{W}=\mathfrak{U} \cup\{003,102,021 U, 021 D, 012\}$, as illustrated with condensed macrostructures of Figure 6(a) shows an initial condensed macrostructure of nodes that are nontrivial strong components of an initial $\mathscr{G}$. (b) The transitive closure of $\mathscr{G}$ turns these strong components into cliques connected by $A^{*}$ relations. This closure contains all permitted types of triads. Figure 6(a) is an example of a gradable weak graph, where each node $i$ can be assigned a grade or level $L_{i}$ in such a way that each arc $i \rightarrow j$ connects the nodes from adjacent levels: $L_{j}=L_{i}+1$. The source of the graph has level 0 , whereas its sink has level 3. All paths between the source and the sink have equal length 3.

Disconnected Condensed Macrostructures. If the graph is disconnected, then each of its connected parts is either strong or unilateral or weak, and all the above applies to each connected part. The classical Cartwright-Harary special case corresponds to a disconnected graph $\mathscr{G}$ with two strong components that transitive closure transforms into cliques.

\section{Discussion}

We discuss the open theoretical problems that our model has addressed, the distinctive properties of the model, and their implications. The modeling of dynamical mechanisms that generate a classic Cartwright and Harary (1956) partition of a group into two mutually antagonistic cliques (parties and factions) has proved to be challenging problem. Such a mechanism must transform an initial network that may include all 16 types of the triad configurations shown in Figure 2, to a 
(a)

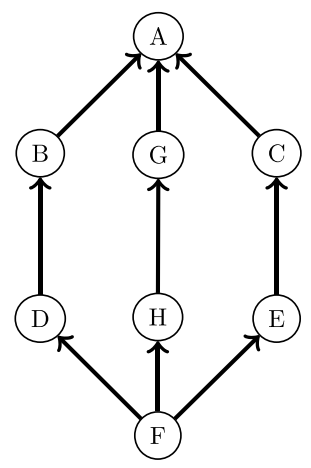

Condensed Macrostructure $\mathscr{G}^{*}$ of $\mathscr{G}$ (b)

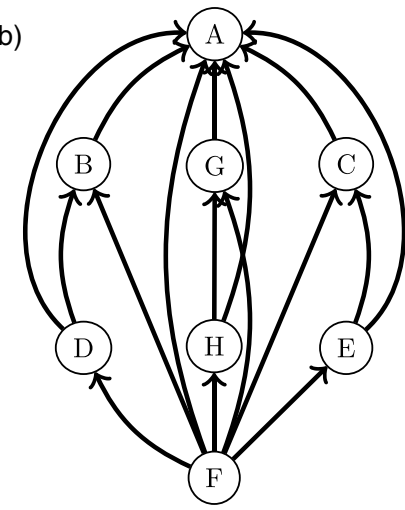

Condensed Macrostructure $\overline{\mathscr{G}}{ }^{*}$ of $\overline{\mathscr{G}}$

Figure 6. (a) is the weak condensed macrostructure of a positive appraisal network and (b) is the condensed macrostructure of its transitive closure. The nodes stand for nontrivial strong components, which in (b) become cliques. The closure contains all nine types of permitted triads $\mathfrak{W}$.

network that includes only two triad types - triads with three symmetric positive relations (the 300 type), and triads with one symmetric positive relation and two symmetric negative relations (the 102 type). Following Kułakowski et al. (2005), a prominent line of work has been advanced under the simplifying assumption of a symmetric initial network (Antal et al., 2006; Marvel et al., 2011; Srinivasan, 2011; Zheng et al., 2014). This assumption eliminates all but 4 of the 16 possible types of triads, and the problem complexity is reduced to a mechanism that eliminates triads with three symmetric negative relations or two symmetric positive relations. Surprisingly, even with this simplification, obtaining a satisfactory dynamical mechanism is unsettled. In generalized balance theory, symmetric relations are an emergent condition restricted to cliques, which might be connected by asymmetric relations. The Cartwright-Harary partition (Cartwright \& Harary, 1956) appears as one special case among others. Generalized balance theory was developed because the empirical evidence on the topology of complete signed networks and their temporal changes does not support the assumption of initial symmetry or the assumption that rules 1-3 (involving enemies) universally constrain individuals' positive and negative relations. The evidence supports the assumption that sign conversions are mainly governed by individuals' eliminations of their violations of transitivity. As we have noted, formulating a dynamical mechanism of individual decision-making that transforms an initial network, including all 16 types of triads, to a transitive network (with no violations of transitivity) is also a challenging problem. In this article, we have presented a solution to this problem based on the assumption of a positive contagion mechanism, which is a special case of the DeGroot (1974) influence system model, in which positive arcs are preserved and propagated by individuals who are resolving their appraisal disagreements with those individuals whom they positively appraise.

Our model also addresses an open problem, posed by generalized balance theory, on the generic macrostructures that result from the evolution of an initial network, which may include all 16 types of triads, to a network with no violations of transitivity. Davis (1967; 1970 ; 1985) defined three types of macrostructures-Classic, Cluster, and Ranked-Clustereach with distinctive set of permitted triad types. Classic balance permits $A=\{300,102\}$, Cluster balance permits $\mathrm{B}=\{A, 003\}$, Ranked-Cluster balance permits $C=\{B, 030 T, 021 U$, $021 D, 120 D, 120 U, 121 D\}$, and the general class of Transitive balance permit $D=\{C, 012\}$, all triads that do not violate transitivity. The definitions of the Cluster and Ranked-Clusters special cases are not derived from principled foundations; for example, Cluster balance relaxes rule 1, but excludes the 012 triad permitted by this relaxation. We have addressed the question of what the 
principled foundation might be. We draw on the fact that any directed network is strongly connected, unilaterally connected, weakly connected, or disconnected. If the network is transitive, then its generic macrostructures must be as follows. If the network is strongly connected, then its set of permitted triads is $\mathfrak{S}=\{300\}$. If the network is unilaterally connected, its set of permitted triads is $\mathfrak{U}=\mathfrak{S} \cup\{120 D, 120 U, 030 T\}$. If the network is weakly connected, then its set of permitted triads is $\mathfrak{W}=\mathfrak{U} \cup\{003,102,021 U, 021 D, 012\}$, which includes all triad types that do not violate transitivity. If network is disconnected, then each of its connected parts is either strong or unilateral or weak, and all the above applies to each connected part. The classical Cartwright-Harary partitioned macrostructure is a special case that appears in the class of disconnected networks, among many other special cases in this class.

We also have addressed the open dynamical system problem of predicting which class of macrostructure a network will evolve to. The network generated by the positive contagion mechanism inherits the connectivity category of the initial network from which it evolved. The strong components of the initial network are the strong components of the evolved network. Any strong component with multiple individuals in the initial network becomes a clique in the evolved network. Any two strong components in the initial network with at least one positive arc connecting them become a complete asymmetric connection in which all members of one clique have positive arcs to all members of the other. A path of such arcs from a source component to a sink component in the macrostructure implies positive asymmetric appraisals of every clique that a clique can reach on that path.

Finally, it may be noted that the postulated contagion mechanism solves an open problem on the application of balance theory to networks that include individuals with a neutral or no appraisal of some other persons, and to large-scale appraisal networks in which the assumption of a complete signed network is unrealistic. The positive contagion mechanism is insensitive to the relaxation of the assumption of a complete signed network. The 16 triad types in Figure 2 may be maintained under the assumption that the missing arcs in them may correspond either to negative, neutral, or no appraisals. No $i \stackrel{ \pm}{\longrightarrow} j$ appraisal can occur if $i$ is unaware of $j$.

The motivation for this contribution is Friedkin's (2006) observation that all the macrostructures of generalized balance theory are interpersonal agreement structures with one or more disjoint sets of structurally equivalent individuals in which the members of each set have identical profiles of appraisals. Every member of a nontrivial clique (i) agrees in the signs of their appraisals of every other member of the network and (ii) has a positive appraisal of every member of their clique. Thus, it is natural to consider a more general definition of balance as such an agreement structure, and employ an interpersonal influence system model (here DeGroot) that is modifying individuals' appraisals of others. Any general influence model must allow for failures to reach balance. Introducing stubborn attachments to negative appraisals would operate to inhibit the evolution to balance. Moreover, an influence system approach raises the possibility that interpersonal appraisals are issue-specific, that is, depending on the issue to a which a group is oriented, individuals' appraisals of particular others may vary.

We conclude with some remarks on the Harary-Cartwright special case of a group in which every individual has a list of friends and enemies and belongs to one of two mutually antagonistic cliques, parties, or factions. If the group is a legislative body, then this special case corresponds to a two-party system in which the expectation of cooperation, agreement, and compromise is low and legislative decisions depend more on the relative sizes of the two parties than on bipartisan deliberative processes. In the U.S. Congress, it took some time and effort to dramatically dampen friendly relations among individuals in different parties, and then to consolidate each party into coherent clique each with little interest in cooperation and compromise. Given the achievement of such a social structure, it will take time and effort to transform it into a social structure that includes a robust set of positive arcs between the parties. Generalized balance theory suggest that any such transformation toward unilateral or weakly connected networks that allow between-clique 
agreements is a transformation toward hierarchically organized influence and control systems that allow agreements.

Acknowledgments. This work was supported by the UC Office of the President through its UC National Laboratory Fees Research Program (Award \#LFR-18-547591), and by a U.S. Army Research Laboratory and the U.S. Army Research Office under grant number W911NF-15-1-0577.

Conflicts of interest. The authors have nothing to disclose.

\section{References}

Abell, P., \& Ludwig, M. (2009). Structural balance: A dynamic perspective. Journal of Mathematical Sociology, 33, $129-155$.

Antal, T., Krapivsky, P. L., \& Redner, S. (2005). Dynamics of social balance on networks. Physical Review E, $72,036121$.

Antal, T., Krapivsky, P. L., \& Redner, S. (2006). Social balance on networks: The dynamics of friendship and enmity. Physica D: Nonlinear Phenomena, 224, 130-136.

Cartwright, D., \& Harary, F. (1956). Structural balance: A generalization of Heider's theory. Psychological Review, 63, 277.

Davis, J. A. (1967). Clustering and structural balance in graphs. Human Relations, 20, 181-187.

Davis, J. A. (1970). Clustering and hierarchy in interpersonal relations: Testing two graph theoretical models on 742 sociomatrices. American Sociological Review, 35, 843-851.

Davis, J. A. (1979). The Davis/Holland/Leinhardt studies: An overview. In Perspectives on Social Network Research, pp. 51-62. Academic Press, New York, London, Toronto, Sydney, San Francisco.

Davis, J. A., \& Leinhardt, S. (1972). The structure of positive interpersonal relations in small groups. In Sociological Theories in Progress. Houghton Miffflin, Boston.

de Rijt, A. Van. (2011). The micro-macro link for the theory of structural balance. The Journal of Mathematical Sociology, 35 , 94-113.

DeGroot, M. H. (1974). Reaching a consensus. Journal of the American Statistical Association, 69, 118-121.

Easley, D., \& Kleinberg, J. (2010). Networks, Crowds and Markets: Reasoning About a Highly Connected World. Cambridge University Press, New York.

Facchetti, G., Iacono, G., \& Altafini, C. (2011). Computing global structural balance in large-scale signed social networks. Proceedings of the National Academy of Sciences, 108, 20953-20958.

Friedkin, N. E. (2004). Social cohesion. Annual Review of Sociology, 30, 409-425.

Friedkin, N. E. (2006). A Structural Theory of Social Influence. Cambridge University Press, New York.

Hallinan, M. T. (1974). A structural model of sentiment relations. American Journal of Sociology, 80, 364-378.

Harary, F., Norman, R. Z., \& Cartwright, D. (1965). Structural Models. An Introduction to the Theory of Directed Graphs. Wiley \& Sons, New York, London, Sydney.

Heider, F. (1944). Social perception and phenomenal causality. Psychological Review, 51, 358-374.

Heider, F. (1946). Attitudes and cognitive organization. The Journal of Psychology, 21, 107-112.

Holland, P. W., \& Leinhardt, S. (1971). Transitivity in structural models of small groups. Comparative Group Studies, 2 , 107-124.

Hummon, N. P., \& Doreian, P. (2003). Some dynamics of social balance processes: Bringing Heider back into balance theory. Social Networks 25, 17-49.

Ilany, A., Barocas, A., Koren, L., Kam, M., \& Geffen, E. (2013). Structural balance in the social networks of a wild mammal. Animal Behaviour, 85, 1397-1405.

Jia, P., Friedkin, N. E., \& Bullo, F. (2016). The coevolution of appraisal and influence networks leads to structural balance. IEEE Transactions on Network Science and Engineering, 3, 286-298.

Johnsen, E. C. (1985). Network macrostructure models for the Davis-Leinhardt set of empirical sociomatrices. Social Networks, 7, 203-224.

Kułakowski, K., Gawroński, P., \& Gronek, P. (2005). The Heider balance: A continuous approach. International Journal of Modern Physics C, 16, 707-716.

Leskovec, J., Huttenlocher, D., \& Kleinberg, J. (2010). Predicting positive and negative links in online social networks. In Proceedings of the 19th International Conference on World Wide Web, pp. 641-650.

Louch, H. (2000). Personal network integration: Transitivity and homophily in strong-tie relations. Social Networks, 22, 45-64.

Marvel, S. A., Kleinberg, J., Kleinberg, R. D., \& Strogatz, S. H. (2011). Continuous-time model of structural balance. Proceedings of the National Academy of Sciences, 108, 1771-1776.

Montgomery, J. D. (2009). Balance theory with incomplete awareness. Journal of Mathematical Sociology, 33, 69-96.

Rawlings, C. M., \& Friedkin, N. F. (2017). The structural balance theory of sentiment networks: Elaboration and test. American Journal of Sociology, 123, 510-548. 
Shils, E. A. (1951). The study of the primary group. In The Policy Sciences, pp. 44-69. Stanford University Press, Stanford.

Sørensen, A. B., \& Hallinan, M. T. (1976). A stochastic model for change in group structure. The Sociological Review, 24, $143-166$.

Srinivasan, A. (2011). Local balancing influences global structure in social networks. Proceedings of the National Academy of Sciences, 108, 1751-1752.

Traag, V. A., Dooren, P. Van, \& Leenheer, P. De. (2013). Dynamical models explaining social balance and evolution of cooperation. Plos One, 8, e60063.

Warshall, S. (1962). A theorem on Boolean matrices. Journal of the ACM, 9, 11-12.

Zheng, X., Zeng, D., \& Wang, F.-Y. (2014). Social balance in signed networks. Information Systems Frontiers, 17, $1077-1095$.

Cite this article: Friedkin N. E., Proskurnikov A. V., and Bullo F. (2019). Positive contagion and the macrostructures of generalized balance. Network Science 7, 445-458. https://doi.org/10.1017/nws.2019.19 